\title{
Aging children of long-lived parents experience slower cognitive decline
}

\author{
Ambarish Dutta $^{\mathrm{a}}$, William Henley ${ }^{\mathrm{b}}$, Jean-Marie Robine ${ }^{\mathrm{c}}$, David Llewellyn ${ }^{\mathrm{a}}$, Kenneth M. Langa ${ }^{\mathrm{d}, \mathrm{e}}$, \\ Robert B. Wallace ${ }^{\mathrm{f}}$, David Melzer ${ }^{\mathrm{a}, *}$ \\ ${ }^{a}$ Epidemiology and Public Health Group, Medical School, University of Exeter, Exeter, UK \\ ${ }^{b}$ PenCLAHRC, Medical School, University of Exeter, Exeter, UK \\ ${ }^{c}$ Institut National de la Sante et de la Recherche Medicale, U710 and U988, Paris and Montpellier, France \\ ${ }^{d}$ Division of General Medicine, University of Michigan Health System, Ann Arbor, MI, USA \\ ${ }^{e}$ VA Ann Arbor Center for Clinical Management Research, Ann Arbor, MI, USA \\ ${ }^{f}$ Center on Aging, Department of Epidemiology, College of Public Health, The University of Iowa, Iowa City, IA, USA
}

\begin{abstract}
Background: Parental longevity confers lower risks for some age-related diseases in offspring. We tested the association between parental longevity and late-life cognitive decline or dementia.

Methods: Data were from the Health and Retirement Study (HRS), a US national sample. Biennial cognitive assessment (Telephone Interview of Cognitive Status-Modified [TICS-m]) occurred for ages 64 years or older in 1996 through 2008 (maximum, 79 years), including physician-diagnosed memory disorder. Offspring were categorized into parental longevity groups based on genderspecific distributional cut points. Model covariates included race, respondents' education, and income status during childhood and adulthood.

Results: Offspring groups did not differ on TICS-m scores at baseline. During follow-up, offspring of two long-lived parents experienced $40 \%$ slower rates of TICS-m decline than those with no longlived parents (95\% confidence interval, $12-72 ; P=.003 ; \mathrm{n}=4731)$. Increased parental longevity was also associated with lower risk of physician-diagnosed memory disorder. Estimates did not change after controlling for environmental variables.

Conclusions: Parental longevity is associated inversely with cognitive decline and self-reported diagnosed memory disorders in aging offspring. Parental longevity may be a valuable trait for identifying early biomarkers for resistance to cognitive decline in aging.

(C) 2014 The Alzheimer's Association. All rights reserved.
\end{abstract}

\section{Introduction}

Longer lived individuals are healthier than their shorter lived counterparts as a result of longevity-promoting intrinsic attributes and favorable environments and health behaviors [1,2]. Children of longer lived parents may experience deceleration of biological aging, longer life, and lower risk of age-related diseases such as diabetes mellitus, cardiovascular disease, and cancer [3] than age-referent control subjects born to parents with shorter longevity [4-8]. This is likely a result of inherited protective factors both intrinsic

\footnotetext{
*Corresponding author. Tel.: +44 139240 6751; Fax: +44 139240 6706.

E-mail address: D.Melzer@exeter.ac.uk
}

and extrinsic from the previous generation. Increased parental life span is also found to be associated with a flatter age-related decline in the domains of physical functioning [9] and activities of daily living [7] in the offspring. As a result, exceptionally long-lived individuals and/or their children are often studied as models of delayed global aging [10].

Cognitive decline in later life is a prominent sign of the biological aging process affecting the brain, and accelerated cognitive decline often marks the preclinical prodrome leading to Alzheimer's disease and other forms of dementia [11]. Although parental longevity is a proxy measure for longevity and the overall aging process in an individual, whether and how it affects cognitive decline and incidence of cognitive impairment and dementia have rarely been studied. A 
previous moderate-size study $(\mathrm{n}=424)$ incorporating a volunteer sample reported a $30 \%$ reduction in cognitive decline in the offspring of long-lived parents [12]. Given the aging worldwide population and our understanding that dementia is one of the most important causes of disability and resource-intense long-term care of the elderly [13], the study of this association may provide new opportunities to identify longevity and cognitive aging "resistance" mechanisms, perhaps including genetic variants through genomewide association studies.

The Health and Retirement Study (HRS) recruited a nationally representative community-dwelling probabilistic sample of 51- to 61-year olds in the United States who have been and are being monitored longitudinally since 1992. This provides us with an opportunity to test the association between parental attained age of HRS participants and their cognitive decline and impairment in a prospective study design.

\section{Methods}

\subsection{Health and Retirement Study}

The HRS recruited a representative sample of Americans older than 50 years using a national area probability sample of U.S. households, with supplemental oversampling of blacks, Hispanics, and residents of the state of Florida. The details of the HRS complex, multistage sampling and survey designs have been documented elsewhere [14]. Briefly, the baseline HRS cohort interviewed age-eligible participants (born between 1931 and 1941, $\mathrm{n}=$ 9763) plus age-ineligible spouses or partners from the selected households. The first wave (baseline) of interviews was conducted in 1992. The repeat interviews were conducted biennially, and the last round of interviews for which the data are available in the public domain was conducted in 2010, thus making it possible for participants to undergo up to 10 waves of interviews if they had not dropped out from the study or died in the interim.

\subsection{Parental attained age}

Respondents were asked at every wave about their parents' age at death or their current age if they were still alive. Parental age at death was calculated from all 10 waves of the study. Of 9763 age-eligible HRS participants 1423 respondents had either one or both parents recorded as living up to their last available records, or had missing records on their status as well as attained age. These respondents were excluded from the analyses.

During our analyses, we used parental longevity as an indicator of the aging process in the parents and hence in the offspring because of its heritability. Consequently, it was necessary to identify "premature" parental deaths, most likely the result of accidents, infection, and reproductive causes, and so forth, which were unrelated to the aging process. We used an empirical approach to characterize the mothers' and fathers' survival in our study population, the details of which have been described elsewhere [3], but are summarized next. The "normal" aging-related human life span is distributed symmetrically around the mode [15] (i.e., the most frequent age at death in later life). To identify prematurely dying parents of HRS participants, we fitted a normal curve using nonlinear least square regression [16] around the modal parental age at death using the following formula:

$$
f=a \times \exp \left[-0.5 \times\left(\frac{x-x 0}{b}\right)^{2}\right]
$$

Because the normal life span varies with gender, these models were fitted separately for mothers and fathers [3]. Based on the results of the normally fitted curves, mothers and fathers who died prematurely (less than the mode less 2 standard deviations [SD]) were identified as those dying before 61 and 46 years respectively [3]. Participants $(\mathrm{n}=1741)$ with parent(s) who died prematurely were excluded from the analyses because these parent(s) did not represent the aging process, which left 6599 participants with valid, ascertained parental life span data. The normally fitted curve around the modal age of parental death was also used to define long-lived mothers and fathers (greater than the mode $+1 \mathrm{SD}$ ), with long-lived mothers being those attaining $91+$ years and long-lived fathers being those attaining $87+$ years. Based on parental longevity, every offspring was assigned one of the three parental longevity ranks: $(i)$ no long-lived parent, (ii) one long-lived parent, and (iii) two long-lived parents.

\subsection{Cognition}

Telephone Interview of Cognitive Status (TICS) was developed by Brandt, Spencer, and Folstein in 1988 [17] for cognitive assessment of survey participants over the telephone by lay interviewers and is based on the Mini-Mental State Examination [18]. The HRS used a reduced version of this test referred to as TICS-Modified (TICS-m) based on the one used by Breitner and colleagues in 1995 $[19,20]$. TICS-m tests multiple domains of cognitive functioning including episodic, working, and semantic memory as well as language, attention, and orientation. Test items include immediate and delayed recall of 10 words, serial seven's subtraction, backward counting from 20 , and naming objects (scissors and cactus), dates (day, month, year, day of the week), and the current president and vice-president [18]. Scores range from $0 \mathrm{pt}$ (worst) to $35 \mathrm{pt}$ (best). The participants who were interviewed through a proxy informant were not offered the TICS-m test.

The TICS-m was administered to all participating HRS respondents during the third wave (1996) for the first time. Then, beginning with the next wave, it was offered biennially - only to participants who were 65 years or older during the interview-through 2008, although some participants with an age of 64 years underwent this test in 1998. At the time of writing, cognitive scores for the 2010 wave are not yet available in the public domain. 
Every age-eligible HRS participant (age, 51-61 years in 1992) was eligible to undergo cognitive testing at least twice by 2008 if they did not die, drop out from the study, miss a cognitive examination, or undergo proxy interviews during any of the intermediate waves.

For our main analyses we included only those respondents with ascertained parental longevity and those who underwent cognitive testing at least twice after attaining $64+$ years $(\mathrm{n}=4731)$, hereafter referred to as "included" participants. Of the included participants, $25 \%$ had two, $22 \%$ had three, $21 \%$ had four, $12 \%$ had five, $10 \%$ had six, and $10 \%$ had seven TICS-m assessments after attaining $64+$ years. The included participants underwent a combined 18,794 TICS-m assessments between 1996 and 2008.

Participants with ascertained parental longevity but less than two cognitive tests after attaining 64+ years $(\mathrm{n}=1868)$ were excluded from the analyses, hereafter referred to as "excluded" participants. Of the excluded participants, 35\% had one and 65\% had no TICS-m assessment after attaining 64+ years. A TICS-m score of less than 9 points was considered cognitive impairment or dementia as described by Wallace and Herzog from the HRS [20].

\subsection{Self-report of physician-diagnosed memory-related diseases}

Beginning with the fourth wave in 1998, HRS participants (as well as proxy informants) were asked during the interview "Has a doctor ever told you that you have memory-related diseases?" as a proxy measure for a cognitive impairment or dementia diagnosis [18]. In subsequent waves, answers from the previous wave(s) to the same question were preloaded as a validation measure.

\subsection{Covariates}

Offspring age was calculated as the years elapsed after attaining age 64, and gender and race were also included as demographic variables in the analyses. Educational achievement as an ordered categorical variable (no education, high school, college, higher/professional degree), and gross income and household wealth as continuous variables in U.S. dollars were indicators of socioeconomic status during adult life of the respondents. Among lifestyle risk factors, smoking (current, ex- and nonsmoker) and body mass index (measured in kilograms per square meter; $<20,20$ $24.9,25-29.9, \geq 30$ ) at baseline were included as ordered categorical variables.

Measures of childhood environment and socioeconomic status of the participants included parental educational achievement in years and fathers' loss of job and consequent receipt of financial help for a considerable period of time.

\subsection{Statistical analyses}

Distribution of baseline characteristics, and prevalence and incidence rate of physician-diagnosed memory disorder across included and excluded samples were explored initially. Then, a distribution of those factors across the three groups of offspring categorized by parental longevity was studied. The summary statistics used were proportion for categorical variables, median with interquartile range for continuous variables with skewed distribution, and mean plus SD for normally distributed continuous variables. The differences in distributions were tested using the $\chi^{2}$ test for categorical variables and the Kruskal-Wallis test or analysis of variance for continuous variables.

Cognitive decline across the three groups of offspring were initially plotted graphically to explore potential differences visually. Linear mixed-effects models with repeat multiwave scores of TICS-m as outcomes were then used to estimate potential differences in cognitive decline. Whether these slopes differed significantly across three groups of offspring was tested formally by introducing an interaction term for age and parental longevity rank in the models. The age-related slopes for different groups of offspring were estimated using stratified analysis. All models were adjusted for race and gender initially (minimally adjusted), and all other environmental factors subsequently (fully adjusted). Three-way interaction among age, parental longevity rank, and gender was tested in the longitudinal models to determine the difference (if any) in cognitive trajectories contingent on gender of offspring.

Cox proportional hazards models were then used to investigate whether the incidence of physician-diagnosed memory disorder varied among the offspring groups. Age at incidence of physician-diagnosed memory disorder during follow-up was the time-to-event outcome. Parental longevity rank was the principal explanatory variable, included initially as a categorical and then as a continuous variable (to test for trend) in the model. Participants who did not experience any event of physician-diagnosed memory disorder but participated through the final wave of the study, or who died or were lost to the follow-up during the intermediate waves were included in the analysis as censored observations. The Cox proportional hazard analysis was conducted on included participants $(n=4646)$, after excluding those with prevalent physician-diagnosed memory disorder at baseline $(\mathrm{n}=21)$, those with missing data for incident events during subsequent waves (truncated observations, $\mathrm{n}=56$ ), and those who experienced incident events before 64 years $(n=8)$. Two hundred five participants in the subsample reported incident physician-diagnosed dementia during follow-up.

Interaction between parental longevity rank and gender of the participants was tested in the Cox models. The assumption of proportional mortality was also tested using Schoenfeld residuals.

The number of participants included in the models is reported in Fig. 1. R statistical package version 2.12.1 was used to analyze the data. Statistical significance was predefined as $P<.05$.

The complex, multistage clustered sampling design was accounted for in the Cox analyses using HRS baseline 


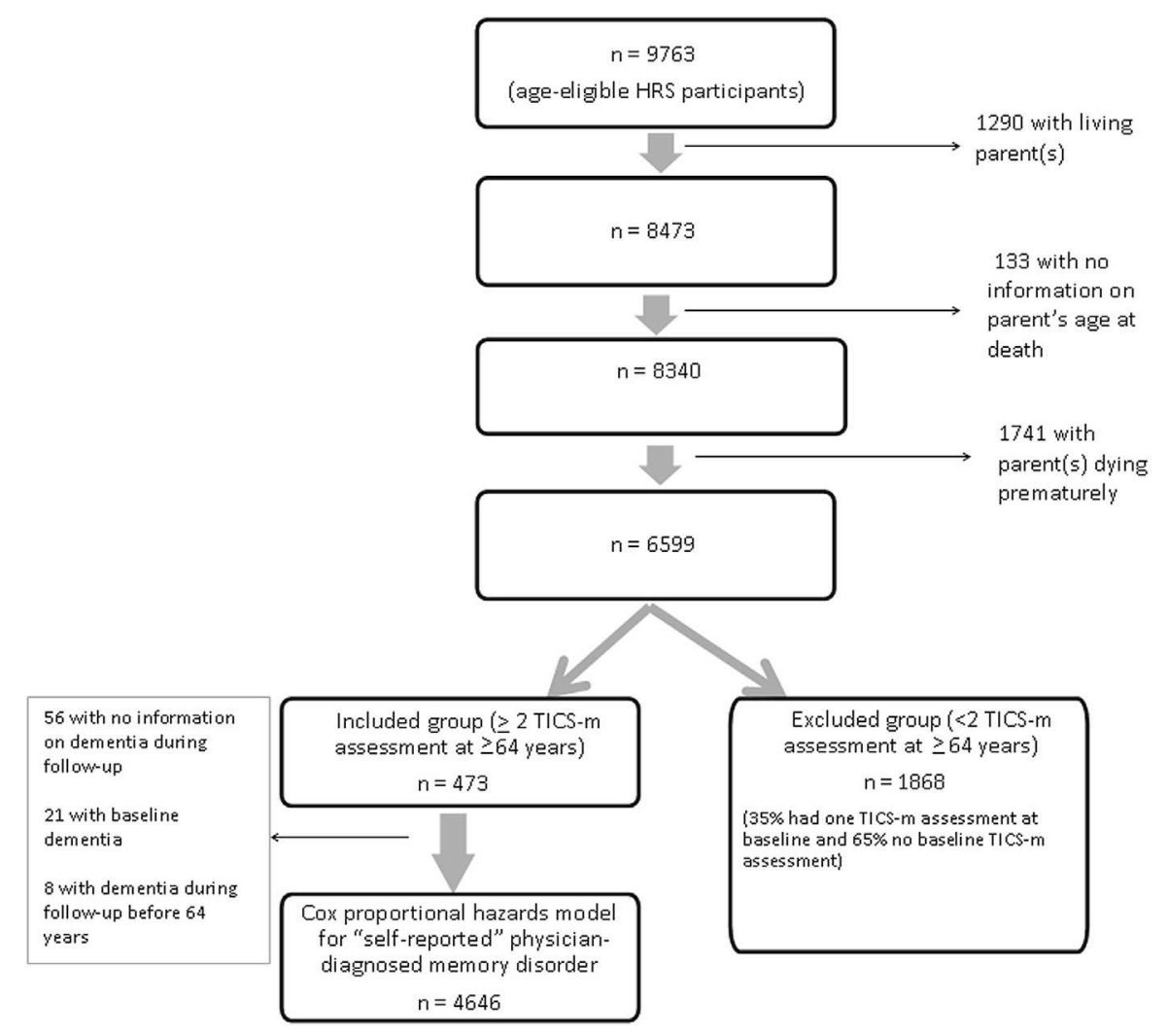

Fig. 1. Health and Retirement Study subsamples included in the analyses. TICS-m, Telephone Interview of Cognitive Status-Modified.

sampling weights and variables for study design. Sampling weights were not used for mixed-effect models because such techniques are problematic [21] and, therefore, perhaps not available in many commonly used statistical software packages for longitudinal analysis (including R), but the longitudinal analyses were adjusted for key weighting-related variables including race.

\section{Results}

The difference between included and excluded groups is presented in Table 1. Excluded participants had less education, wealth, and income than the included sample. They also reported higher baseline as well as higher incidence rates of physician-diagnosed memory disorder. They experienced higher death and dropout rates than those included in the study. A smaller proportion of them had long-lived parents (30\% of the included sample, with one or two long-lived parents, compared with $19 \%$ in the excluded group; $P<.001)$. The average baseline TICS-m score at $64+$ years of the excluded participants with one TICS-m assessment ( $\mathrm{n}=648$ ) was significantly less than that of the included sample $(\mathrm{n}=4731$; TICS- $\mathrm{m}$ score in excluded group mean, $21.72 \pm 5.38$ points [SD]; included group mean, $23.58 \pm 4.67$ points; $P<.001$ ).

We then compared the baseline characteristics across three groups of offspring included in the main analyses
(Table 2). The offspring with long-lived parents were more financially affluent. They were better educated (33\% college graduate or higher in group 3 vs. $21 \%$ in group $1, P<.001$ ), less overweight or obese (56\% in group 3 vs. $60 \%$ in group $1, P=.003$ ), and had greater household income and wealth (US\$ 135,000 in group 3 vs. US\$ 94,000 in group 1, $P<.001)$. They also had slight socioeconomic advantages during their childhood, measured by any prolonged unemployment faced by fathers (17\% in group 1 vs. $20 \%$ in group $3, P=.05)$ and by their family forced to receive financial help to tide over difficult situations (8\% in group 1 vs. $11 \%$ in group $3, P=.04)$. These differentials in environmental risk factors, although modest, showed a monotonous trend across the three groups.

The groups did not differ in baseline age at which TICS$m$ was first administered or the numbers of TICS- $m$ assessments conducted during follow-up.

In the longitudinal mixed-effects model with repeat measures of cognition, the TICS-m score at age 64+ (intercept) did not differ significantly $(P=.1)$. However, the rate of $\operatorname{cog}$ nitive decline after $64+$ years was significantly different (Fig. 2) in the three groups categorized by parental longevity ( $P$ for interaction $=.003)$. The per-year decline in TICS-m score after $64+$ years was 0.24 point (range, $0.22-0.26$ point) in participants with no long-lived parent, 0.20 point (range, $0.17-0.23$ point) in those with one long-lived parent, and 0.14 point (range, $0.04-0.24$ point) in those with two 
Table 1

Description of included and excluded Health and Retirement Study participants in the studied subsample

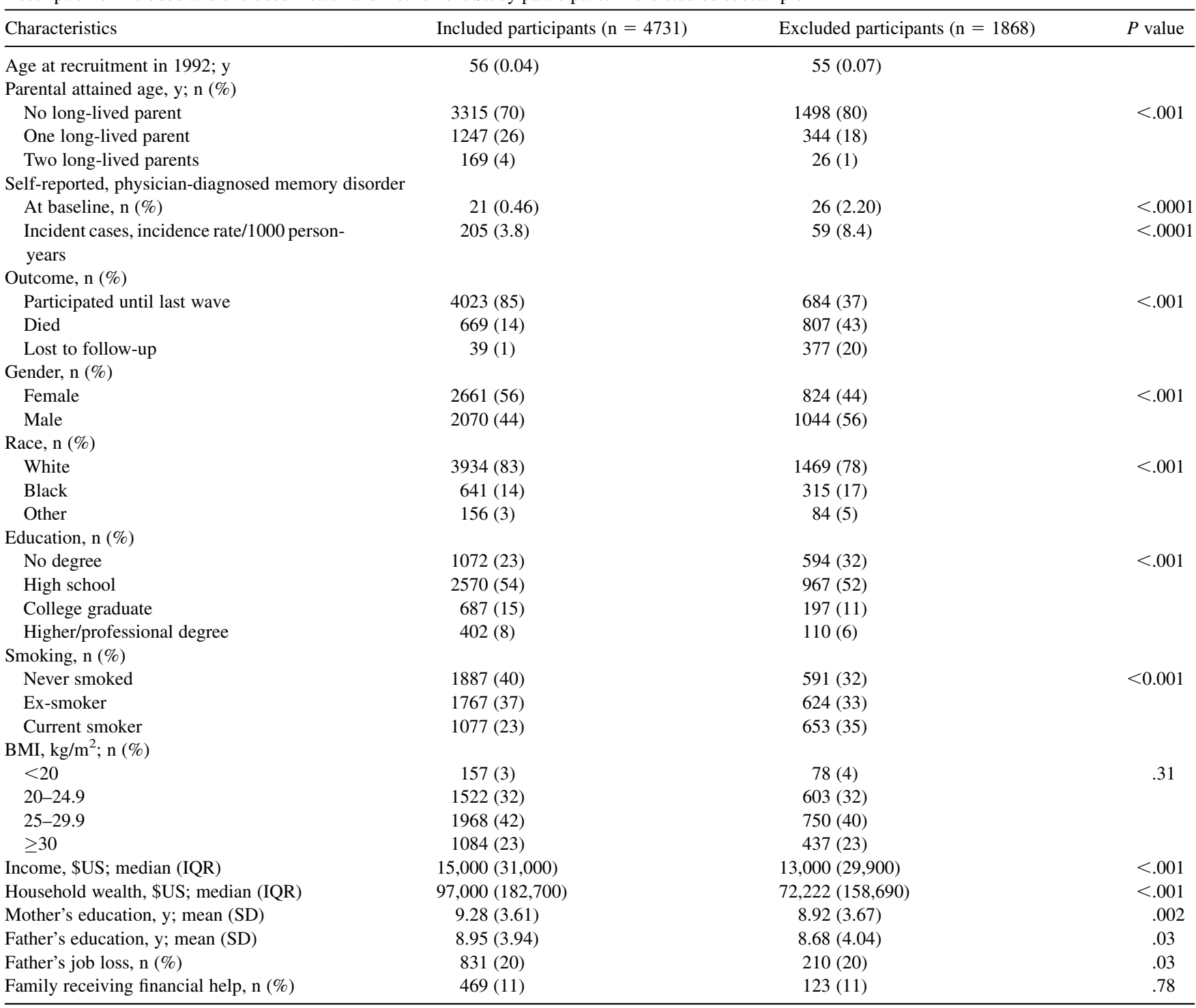

Abbreviations: BMI, body mass index; IQR, interquartile range; $\mathrm{SD}$, standard deviation.

long-lived parents $(P$ for group differences $=.003)$. These estimates did not change after adjustment for environmental variables (Table 3). The three-way interaction term among age, parental longevity rank, and gender of the offspring was not significant.

Cox proportional hazard regression showed a declining trend in the risk of physician-diagnosed memory disorder with an increase in the number of long-lived parents. The estimates changed only marginally between the basic and fully adjusted models. The fully adjusted hazard ratio was 0.71 (range, 0.53-0.96) in participants with one long-lived parent and 0.58 (range, $0.24-1.40$ ) in those with two long-lived parents $(P$ for trend $=.02)$.

The interaction term for gender and parental life span was not significant, and the assumption for proportional hazards was not violated in the Cox models.
The following sensitivity analyses were carried out which are described below:

There were 34 participants in the included sample with TICS-m score of less than 9 points at baseline (64+ years). Exclusion of these participants from the mixed-effects models did not alter the estimates for cognitive trajectories. Similarly, exclusion of participants $(n=21)$ with baseline self-reported, physician-diagnosed memory disorder did not alter the pattern of associations.

Subjects with at least one living parent were excluded from the final analysis $(n=1290)$. We conducted an additional analysis including participants with living parent(s) who also underwent at least two TICS-m assessments after 64 years of age $(n=391)$. The per-year decline in TICS-m score after $64+$ years was 0.25 point (range, $0.17-0.34$ points) in that group, which is comparable with the 
Table 2

Baseline characteristics of Health and Retirement Study offspring categorized by parental longevity

\begin{tabular}{|c|c|c|c|c|}
\hline Characteristics & No long-lived parent $(\mathrm{n}=3315)$ & One long-lived parent $(\mathrm{n}=1247)$ & Two long-lived parents $(\mathrm{n}=169)$ & $\begin{array}{l}P \\
\text { value }\end{array}$ \\
\hline Age at first TICS-m assessment, $y$ & $65(1)$ & $65(1)$ & $65(1)$ & .8 \\
\hline No. of TICS-m assessments & $4(3)$ & $4(2)$ & $4(2)$ & .17 \\
\hline Female & $1410(43)$ & $588(47)$ & $72(43)$ & \multirow[t]{2}{*}{.03} \\
\hline Male & $1905(57)$ & $659(53)$ & $97(57)$ & \\
\hline \multicolumn{5}{|l|}{ Race, n (\%) } \\
\hline Other & $99(3)$ & $50(4)$ & $7(4)$ & .16 \\
\hline \multicolumn{5}{|l|}{ Education, $\mathrm{n}(\%)$} \\
\hline No degree & $790(24)$ & $259(21)$ & $31(18)$ & \multirow[t]{4}{*}{$<.001$} \\
\hline High school & $1823(55)$ & $665(53)$ & $82(49)$ & \\
\hline College graduate & $445(13)$ & $208(16)$ & $34(20)$ & \\
\hline Higher/professional degree & $257(8)$ & $123(10)$ & $22(13)$ & \\
\hline \multicolumn{5}{|l|}{ BMI, $\mathrm{kg} / \mathrm{m}^{2} ; \mathrm{n}(\$)$} \\
\hline$<20$ & $106(3)$ & $40(3)$ & $11(7)$ & \multirow[t]{4}{*}{.003} \\
\hline $20-24.9$ & $1058(32)$ & $407(33)$ & $57(34)$ & \\
\hline $25-29.9$ & $1344(41)$ & $556(44)$ & $68(40)$ & \\
\hline$\geq 30$ & $807(24)$ & $244(20)$ & $33(19)$ & \\
\hline Income, \$US; median (IQR) & $15,000(30,000)$ & $17,200(32,600)$ & $16,000(31,500)$ & .03 \\
\hline Household wealth, \$US; median (IQR) & $94,000(174,400)$ & $100,000(198,500)$ & $135,500(254,000)$ & $<.001$ \\
\hline Mother's education, y; mean (SD) & $9(4)$ & $9(5)$ & $10(5)$ & .34 \\
\hline Father's education, y; mean (SD) & $8(6)$ & $8(6)$ & $8(6)$ & .43 \\
\hline Father's job loss, n (\%) & $587(20)$ & $217(19)$ & $27(17)$ & .05 \\
\hline Family receiving financial help, n (\%) & $350(11)$ & $106(9)$ & $13(8)$ & .04 \\
\hline $\begin{array}{l}\text { Average TICS-m score at baseline with age } \\
\geq 64 \mathrm{y}, \mathrm{pt}\end{array}$ & 23.92 & 24.04 & 24.48 & .1 \\
\hline
\end{tabular}

Abbreviations: TICS-m, Telephone Interview of Cognitive Status-Modified; BMI, body mass index; IQR, interquartile range; SD, standard deviation.

NOTE. There were 260 participants with missing data for family receiving help, 566 participants with missing data for father losing job, 297 participants with missing data for mother's education, and 461 participants with missing data for father's education.

estimates of offspring born of two long-lived parents in the main analysis.

\section{Discussion}

In this large, U.S. population-based prospective study, we examined the relationship between parental life span and measures of cognitive aging in HRS participants attaining at least 64 years and monitored to a maximum of 79 years of age. We found that offspring age 64 and older with long-lived parents had a slower rate of cognitive decline and a reduced risk of physician-diagnosed memory disorder over 12 years (mean follow-up period, 6.1 years). There was evidence of a monotonic relationship, and the association remained significant after adjusting for environmental variables. Excluding participants with cognitive impairment or dementia at baseline did not change the pattern of associations. To our knowledge, this is the first large-population representative study to demonstrate that having long-lived parents is associated with a slower rate of measured cognitive decline and a reduced risk of physician-diagnosed memory dysfunction, as reported by the respondents.
Long-lived parents have been reported to confer survival benefits and protection from chronic diseases to their offspring [10,22-25], such as diabetes mellitus, hypertension, and cardiovascular diseases, than control subjects $[23,26]$. This familial longevity advantage and disease resistance may be a combination of inherited environmental factors and intrinsic attributes. Our study has shown evidence of a modest association between parental longevity and environmental factors, signifying that long-lived parents may pass on to their children some favorable socioeconomic and lifestyle factors, although controlling for these environmental variables modified the parental longevity effect on cognitive decline or incident memory disorder only to a small extent. This, perhaps, suggests that this protective effect of parental longevity is mediated primarily by inherited biological attributes.

To our knowledge, only one previous study has tested the association between parental life span and cognitive aging, reporting 30\% less cognitive decline among offspring born to long-lived parent(s). That study recruited a smaller volunteer sample $(\mathrm{n}=424)$ of $75+$-year-old offspring born to long-lived parent(s) [12], and parental longevity was defined by life expectancy-based gender-neutral cut points. 


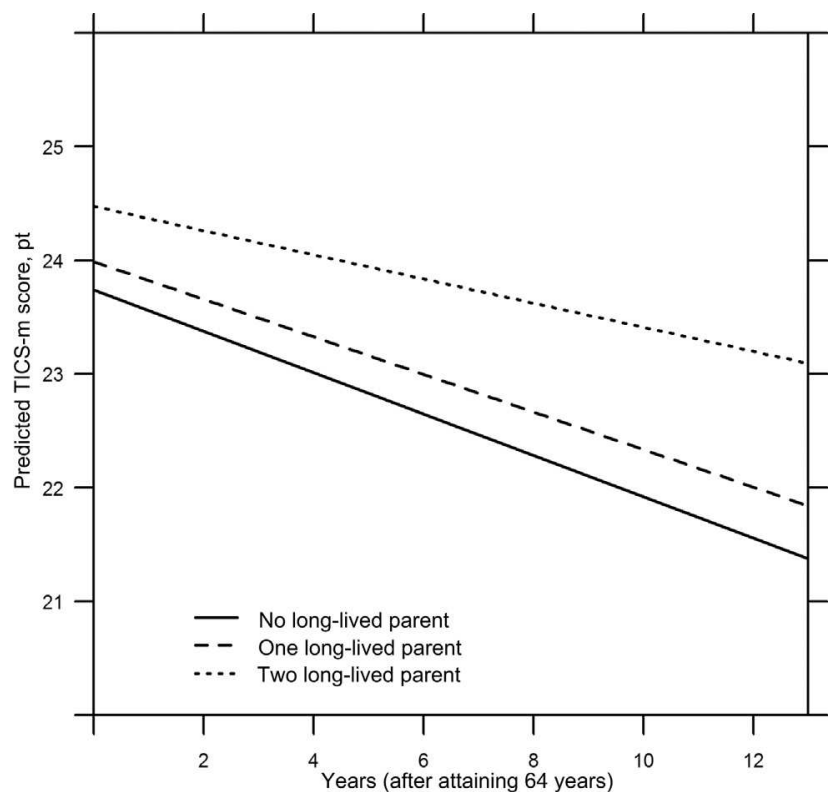

Fig. 2. Trajectory of Telephone Interview of Cognitive Status-Modified (TICS-m) score after attaining 64 years in three groups of Health and Retirement Study offspring categorized by parental longevity $(n=4731)$.

The limitations of our study include lack of clinical diagnosis of dementia, and self-report of physiciandiagnosed memory disorders as a proxy of cognitive impairment and dementia diagnosis may be prone to underreporting and misclassification in a survey sample. However, it is unlikely that such misclassification is distributed differentially across groups of offspring categorized by their parental longevity ranks. Validation of the findings in an independent sample with clinical diagnoses established systematically or in clinical records would strengthen the evidence.

The slope of age-related decline in cognition was estimated without sampling weights because inclusion is technically problematic [21], and, perhaps as a result, is not available in $\mathrm{R}$ software, but models were adjusted for race, a key weighting variable.
Another limitation is sample attrition, as experienced by most longitudinal studies. The excluded sample in our study had higher death and dropout rates than the included sample, which was the reason why they could not be tested with TICS-m at least twice and qualified for exclusion. Those excluded are likely to have experienced lower overall TICS-m scores and perhaps a steeper decline with age than the included sample, as evident by significantly lower baseline TICS-m scores, and higher prevalent and incident memory disorder rates. Because the excluded sample members are also less likely to be born of long-lived parent(s), their exclusion from the analysis has perhaps underestimated the effects of parental longevity on cognitive decline.

Another limitation of the study was unavailability of the age of death of some parent(s), although the rate of ascertainment of parental death was much higher than other comparable studies (described later).

The strengths of our study include a large, nationally representative population sample of HRS participants monitored for 12 years, and examined for cognitive status four times, on average. Another strength of our study is that the data on parental death were collected longitudinally during offspring interviews, which helped to ascertain the mortality status of $87 \%$ of the parents. In previous studies, almost half the parents were still alive at baseline and were not monitored further [25]. Another strength of our study is the use of the gender-specific, empirically determined cut points to define parents dying prematurely or living a long time, in contrast to several other studies in this area of research that used arbitrary cut points $[7,8,12]$.

In conclusion, we found that offspring of long-lived parents aged 64 years and older experienced a reduced rate of cognitive decline and a lower risk of memory dysfunction over 12 years. This effect was attenuated marginally by accounting for environmental factors. Future studies are therefore warranted to investigate the biological markers and mechanisms that may confer this advantage from having long-lived parents, and this may lead ultimately to new possibilities for the prevention of cognitive decline and dementia.

Table 3

Cognitive decline and risk (hazard ratio) of self-reported, physician-diagnosed memory disorder across the three groups of HRS offspring categorized by parental longevity

\begin{tabular}{llll}
\hline Outcomes & No long-lived parent & One long-lived parent & Two long-lived parents \\
\hline $\begin{array}{l}\text { Cognitive decline after } \geq 64 \mathrm{y} \\
\quad \text { value for interaction }\end{array}$ & $\mathrm{n}=3315$ & $\mathrm{n}=1247$ & $\mathrm{n}=169$ \\
$\quad \begin{array}{l}\text { Minimally adjusted model* } \\
\quad \text { Fully adjusted model }\end{array}$ & $0.24(0.23-0.26)$ & $0.2(0.17-0.23)$ & $0.14(0.05-0.23)$ \\
$\begin{array}{l}\text { Physician-diagnosed memory disorder } \\
\quad \text { value for trend }\end{array}$ & $0.24(0.23-0.26)$ & $0.19(0.16-0.23)$ & $0.14(0.04-0.23)$ \\
$\quad \mathrm{n}=3251$ & $\mathrm{n}=1228$ & $\mathrm{n}=167$ & .003 \\
$\quad \begin{array}{l}\text { Minimally adjusted model } \\
\text { Fully adjusted model }\end{array}$ & 1 & $0.69(0.51-0.93)$ & $0.58(0.24-1.43)$ \\
\end{tabular}

Abbreviations: HRS, Health and Retirement Study.

*Adjusted for race and gender.

${ }^{\dagger}$ Adjusted for race, gender, education, income, household wealth, smoking, body mass index, parental educational achievement, fathers' job loss, and family receiving financial help. 


\section{Acknowledgments}

We thank all the participants of the HRS. Funding for this analysis was from internal University of Exeter resources.

\section{RESEARCH IN CONTEXT}

1. Systematic review: We reviewed the literature in databases including PubMed using the terms "offspring," "children of long-lived parents," "centenarians," "nonagenarians," and "cognition" in various combinations. We found only one publication that examined the association between parental longevity and cognitive trajectories or cognitive impairments (in a volunteer sample of offspring $[n=424]$ ), which has been cited and discussed in the manuscript.

2. Interpretation: Our analysis has shown that parental longevity is associated negatively with cognitive decline in a sample of older Americans. This result is consistent with the existing knowledge of parental longevity being protective against several common age-related diseases in the offspring.

3. Future directions: We suggest the findings of the study may be partially taken forward partly through studies of genomic and other biomarkers in offspring categorized by their parental longevity. Studies of biomarkers before cognitive differences become apparent might provide useful predictors of the causal processes. Such studies may help to identify the biological attributes of resistance or vulnerability to brain aging before established brain pathologies obscure the underlying drivers.

\section{References}

[1] Galioto A, Dominguez LJ, Pineo A, Ferlisi A, Putignano E, Belvedere M, et al. Cardiovascular risk factors in centenarians. Exp Gerontol 2008;43:106-13.

[2] Perls TT, Wilmoth J, Levenson R, Drinkwater M, Cohen M, Bogan H, et al. Life-long sustained mortality advantage of siblings of centenarians. Proc Natl Acad Sci U S A 2002;99:8442-7.

[3] Dutta A, Henley W, Robine JM, Langa KM, Wallace RB, Melzer D. Longer lived parents: protective associations with cancer incidence and overall mortality. J Gerontol A Biol Sci Med Sci 2013 May 16. [Epub ahead of print].

[4] Terry DF, Wilcox M, McCormick MA, Lawler E, Perls TT. Cardiovascular advantages among the offspring of centenarians. J Gerontol A Biol Sci Med Sci 2003;58:M425-31.

[5] Vandenbroucke JP, Matroos AW, Van der Heide-Wessel C, Van der Heide RM. Parental survival, an independent predictor of longevity in middle-aged persons. Am J Epidemiol 1984;119:742-50.
[6] Abbott MH, Abbey H, Bolling DR, Murphy EA. The familial component in longevity:- a study of offspring of nonagenarians: III. Intrafamilial studies. Am J Med Genet 1978;2:105-20.

[7] Gjonca E, Zaninotto P, Gjonça E. Blame the parents? The association between parental longevity and successful ageing. Demogr Res 2008; 19:1435-50.

[8] Ikeda A, Iso H, Toyoshima H, Kondo T, Mizoue T, Koizumi A, et al. Parental longevity and mortality amongst Japanese men and women: the JACC Study. J Intern Med 2006;259:285-95.

[9] Klein BEK, Knudtson MD, Lee KE, Klein R. Parents' attained age and biomarkers of aging in their children. Arch Gerontol Geriatr 2009; 49:284-8.

[10] Westendorp RGJ, Van Heemst D, Rozing MP, Frölich M, Mooijaart SP, Blauw G-J, et al. Nonagenarian siblings and their offspring display lower risk of mortality and morbidity than sporadic nonagenarians: the Leiden Longevity Study. J Am Geriatr Soc 2009; $57: 1634-7$.

[11] Wilson RS, Leurgans SE, Boyle PA, Bennett DA. Cognitive decline in prodromal Alzheimer disease and mild cognitive impairment. Arch Neurol 2011;68:351-6.

[12] Lipton RB, Hirsch J, Katz MJ, Wang C, Sanders AE, Verghese J, et al. Exceptional parental longevity associated with lower risk of Alzheimer's disease and memory decline. J Am Geriatr Soc 2010; 58:1043-9.

[13] Brookmeyer R, Johnson E, Ziegler-Graham K, Arrighi HM. Forecasting the global burden of Alzheimer's disease. Alzheimers Dement 2007;3:186-91.

[14] HRS project staff. Sample sizes, response rates and mortality ascertainment, 2011. Available at: http://hrsonline.isr.umich.edu/sitedocs/ sampleresponse.pdf. Accessed August 30, 2013.

[15] Kannisto V. Mode and dispersion of the length of life. Population 2001;13:159-71.

[16] Cheung SLK, Robine J-M. Increase in common longevity and the compression of mortality: the case of Japan. Population Stud 2007; 61:85-97.

[17] Brandt J, Spencer M, Folstein M. The Telephone Interview for Cognitive Status. Cogn Behav Neurol 1988;1:111-7.

[18] Wallace RB, Weir DR, Langa KM, Faul JD. HRS/AHEAD documentation report: documentation of cognitive functioning measures in the Health and Retirement Study. University of Michigan, Ann Arbor, MI; 2005.

[19] Breitner JC, Welsh KA, Gau BA, McDonald WM, Steffens DC, Saunders AM, et al. Alzheimer's disease in the National Academy of Sciences-National Research Council Registry of Aging Twin Veterans. III. Detection of cases, longitudinal results, and observations on twin concordance. Arch Neurol 1995;52:763-71.

[20] Herzog AR, Wallace RB. Measures of cognitive functioning in the AHEAD Study. J Gerontol A Biol Sci Med Sci 1997;52:37-48.

[21] Gelman A. Struggles with survey weighting and regression modeling. Stat Sci 2007;22:153-64.

[22] Frederiksen H, McGue M, Jeune B, Gaist D, Nybo H, Skytthe A, et al. Do children of long-lived parents age more successfully? Epidemiology (Cambridge, Mass) 2002;13:334-9.

[23] Terry DF, Evans JC, Pencina MJ, Murabito JM, Vasan RS, Wolf PA, et al. Characteristics of Framingham offspring participants with long-lived parents. Arch Intern Med 2007;167:438-44.

[24] Adams ER, Nolan VG, Andersen SL, Perls TT, Terry DF. Centenarian offspring: start healthier and stay healthier. J Am Geriatr Soc 2008; 56:2089-92.

[25] Rosengren A, Thelle D, Wilhelmsen L. Parental age and coronary disease in the general male population. J Intern Med 2002; 251:258-67.

[26] Terry DF, Wilcox MA, McCormick MA, Perls TT. Cardiovascular disease delay in centenarian offspring. J Gerontol A Biol Sci Med Sci 2004:59:385-9. 